\title{
Retrospective study comparing MR-guided radiation therapy (MRgRT) setup strategies for prostate treatment: repositioning vs. replanning
}

Jung-in Kim ${ }^{1,2,3}$, Jong Min Park ${ }^{1,2,3,4}$, Chang Heon Choi ${ }^{1,2,3}$, Hyun Joon An ${ }^{1,2,3}$, Yi-Jun Kim ${ }^{1}$ and Jin Ho Kim ${ }^{1,2,3^{*}}$

\begin{abstract}
Background: This study compared adaptive replanning and repositioning corrections based on soft-tissue matching for prostate cancer by using the magnetic resonance-guided radiation therapy (MRgRT) system.

Methods: A total of 19 patients with prostate cancer were selected retrospectively. Weekly magnetic resonance image (MRI) scans were acquired for 5 weeks for each patient to observe the anatomic changes during the treatment course. Initial intensity-modulated radiation therapy (IMRT) plans (ilMRT) were generated for each patient with 13 coplanar ${ }^{60} \mathrm{Co}$ beams on a ViewRay ${ }^{\mathrm{TM}}$ system. Two techniques were applied: patient repositioning and replanning. For patient repositioning, one plan was created: soft-tissue (prostate) matching (Soft). The dose distribution was calculated for each MRI with the beam delivery parameters from the initial IMRT plan. The replanning technique was used to generate the Adaptive plan, which was the reoptimized plan for the weekly MRI. The dose-volumetric parameters of the planning target volume (PTV), bladder, and rectum were calculated for all plans. During the treatment course, the PTV, bladder, and rectum were evaluated for changes in volume and the effect on dosimetric parameters. The differences between the dose-volumetric parameters of the plans were examined through the Wilcoxon test. The initial plan was used as a baseline to compare the differences.
\end{abstract}

Results: The Adaptive plan showed better target coverage during the treatment period, but the change was not significant in the Soft plan. There were significant differences in $\mathrm{D}_{98 \%}, \mathrm{D}_{95 \%}$, and $\mathrm{D}_{2 \%}$ in PTV between the Soft and Adaptive plans $(p<0.05)$ except for $D_{\text {mean. }}$. There was no significant change in $D_{\max }$ and $D_{\text {mean }}$ as the treatment progressed with all plans. All indices for the Adaptive plan stayed the same compared to those of ilMRT during the treatment course. There were significant differences in $D_{15 \%}, D_{25 \%}, D_{35 \%}$, and $D_{50 \%}$ in the bladder between the Soft and Adaptive plans. The Adaptive plan showed the worse dose sparing than the Soft plan for the bladder according to each dosimetric index. In contrast to the bladder, the Adaptive plan achieved better sparing than the Soft plan during the treatment course. The significant differences were only observed in $D_{15 \%}$ and $D_{35 \%}$ between the Soft and Adaptive plans $(p<0.05)$.

Conclusions: Patient repositioning based on the target volume (Soft plan) can relatively retain the target coverage for patients and the OARs remain at a clinically tolerance level during the treatment course. The Adaptive plan did not clinically improve for the dose delivered to OARs, it kept the dose delivered to the target volume constant. However, the Adaptive plan is beneficial when the organ positions and volumes change considerable during treatment.

Keywords: Soft-tissue repositioning, Adaptive replanning, Prostate radiotherapy, MRI-guided treatment

\footnotetext{
* Correspondence: jinho.kim.md@gmail.com

'Department of Radiation Oncology, Seoul National University Hospital,

Seoul, Republic of Korea

${ }^{2}$ Biomedical Research Institute, Seoul National University Hospital, Seoul,

Republic of Korea

Full list of author information is available at the end of the article
}

(c) The Author(s). 2019 Open Access This article is distributed under the terms of the Creative Commons Attribution 4.0 International License (http://creativecommons.org/licenses/by/4.0/), which permits unrestricted use, distribution, and reproduction in any medium, provided you give appropriate credit to the original author(s) and the source, provide a link to the Creative Commons license, and indicate if changes were made. The Creative Commons Public Domain Dedication waiver (http://creativecommons.org/publicdomain/zero/1.0/) applies to the data made available in this article, unless otherwise stated. 


\section{Background}

The goal of radiation therapy is to deliver an accurate prescription dose to the target while minimizing the dose to normal tissue. External beam radiotherapy (EBRT) is a common definitive treatment option for localized prostate cancer [1-7]. In the last decade, significant technological advances in EBRT have been achieved. Intensity-modulated radiation therapy (IMRT) is a type of conformal radiation therapy and is widely used to treat prostate cancer $[1,7,8]$. However, the complexity of the treatment delivery and inter- and intra-fraction variations are a concern when using IMRT for prostate cancer $[9,10]$. Adding margins around the clinical target volume (CTV) can account for these variations to ensure target coverage. Gill et al. reported that when daily cone beam computed tomography (CBCT) was used for soft-tissue alignment of the prostate, a PTV margin of $3 \mathrm{~mm}$ allowed sufficient coverage for CTV [11]. On the other hand, in Engels's study, margins of $6 \mathrm{~mm} \mathrm{LR}$ and $10 \mathrm{~mm} \mathrm{AP}$ and $\mathrm{CC}$ were used in patients who did not hve markers implanted in them. Margins of $3 \mathrm{~mm}$ LR and $5 \mathrm{~mm} \mathrm{AP}$ and $\mathrm{CC}$ were used for patients with implanted markers [12]. However, expanding the irradiated volume consequently increases normal organ toxicity, which is limited by the tolerances of the bladder and rectum $[8,13]$. In recent years, advances in image guidance have allowed for better localization of the prostate [14-20]. Using image guidance together with IMRT delivers the dose to the prostate more precisely with smaller margins to decrease the doses to the rectum and bladder. Image-guided radiation therapy (IGRT) technology has been widely adopted to provide anatomic information with the patient in the treatment position. With image guidance (typically X-ray imaging such as kilovolt $(\mathrm{kV})$, megavolt (MV), and CBCT), the patient positions in the daily images are registered to the planning images based on either bony landmarks or soft tissues [16-18, 21, 22]. Furthermore, fiducial markers for prostate IGRT have been in use since the 1990s and O'Neill et al. reviewed the evidence for the use of fiducial markers in clinical practice [23]. Image guidance corrects not only for patient position variations but also for changes in the target volume and shapes. Adaptive radiation therapy (ART) has been introduced to compensate for variations in patient treatment during a radiotherapy course [24-26]. ART techniques can be categorized as offline or online adaptive planning [27]. Offline adaptive planning uses a feedback strategy for current treatment by incorporating the obtained daily treatment images. This adaptive planning reflects patient-specific anatomic variations and provides a reoptimized treatment plan for the remaining treatment fractions. Online adaptive planning is the optimal strategy for each fraction delivery because the treatment plan is reoptimized while the patient is waiting on the treatment table. However, clinical implementation of both offline and online adaptive techniques with a common imaging modality based on X-rays is still in the development phase because it is not sufficient to modify the treatment plan according to anatomic changes on inter- and intra-fraction bases. In 2009, Thongphiew et al. compared three online IGRT techniques (bony-anatomy matching, soft-tissue matching, and online replanning) for prostate IMRT treatment using daily CBCT [18]. They demonstrated that $\mathrm{CBCT}$ can feasibly be used for reoptimizing the treatment plan online, which has significant benefits when a high degree of deformation or differential organ position displacement occurs. Clinical implementation of ART for prostate cancer has been greatly promoted by image guidance with onboard CBCT [28-31]. Onboard CBCT allows the target volume and adjacent anatomies to be localized before treatment of a patient in the treatment position. However, CBCT has insufficient image quality compared to planning computed tomography (CT) to provide an accurate delineation of structures and dose calculation owing to the higher noise and lower contrast $[29,32]$. Furthermore, the inconsistency between the CT number to electron density curves of $\mathrm{CT}$ and $\mathrm{CBCT}$ images can result in a discrepancy in the dose calculations.

Recently, the high soft-tissue contrast and real-time imaging capability of magnetic resonance imaging (MRI) have allowed for more accurate assessment of inter- and intra-fraction variations [33-36]. MR-guided radiotherapy (MRgRT) systems that integrate MR scanners with radiation delivery machines can potentially facilitate the online ART strategy. ViewRay ${ }^{\text {Tm }}$ (ViewRay Inc., Cleveland, OH, USA) is the first commercial MRgRT system and has been treating patients since 2014 [37]. Much work has been carried out on prostate treatment with the MRgRT system because it is very useful for identifying the prostate as well as detecting normal tissues [33, 34, 38-40]. The present study compared adaptive replanning and repositioning corrections based on soft-tissue matching for prostate cancer with the ViewRay system in a retrospective manner.

\section{Methods}

\section{Patient data and study design}

After institutional review board approval, a total of 19 patients with prostate cancer were selected for this study in a retrospective manner. Low-risk patients did not receive androgen deprivation therapy (ADT). Intermediate and high risk patients received neoadjuvant ADT. Table 1 summarizes the patients' clinical characteristics. All patients were planned with IMRT using ViewRay. A CT image set and MRI image set of each patient in the supine position were acquired once before treatment. The Brilliance Big Bore ${ }^{\text {Tu }} \mathrm{CT}$ simulator (Philips, Cleveland, OH, USA) was used for CT scanning with a slice thickness of $1.5 \mathrm{~mm}$. Each patient was immobilized with Smart Rest ${ }^{\mathrm{m}}$ (Chunsung, Seoul, Republic of Korea), which combines 
Table 1 Summary of patients' clinical characteristics

\begin{tabular}{lll}
\hline Age (years) & Mean (range) & $77(65-86)$ \\
\hline T stage & T1 & $2(10.5 \%)$ \\
& T2 & $11(57.9 \%)$ \\
T3 & $2(10.5)$ \\
GS score & T4 & $4(21.1 \%)$ \\
& $6(3+3)$ & $3(15.8 \%)$ \\
& $7(3+4)$ & $3(15.8 \%)$ \\
& $7(4+3)$ & $5(26.3 \%)$ \\
& $8(4+4)$ & $7(36.8 \%)$ \\
aPSA & $9(4+5)$ & $1(5.3 \%)$ \\
& Median (range) & $18.89(2.19-161.95)$ \\
bADT use & Mean (SD) & $27.78(35.63)$ \\
\hline
\end{tabular}

${ }^{\mathrm{a}} P S A$ prostate-specific antigen; ${ }^{\mathrm{b}} A D T$ androgen deprivation therapy

the knee fix and feet fix. The initial CT image (iCT) sets were only used to calculate the dose for the MRIdian ${ }^{\text {mix }}$ system (ViewRay Inc., Cleveland, OH, USA); no CT images were taken during treatment. Initial MRI (iMRI) was used to draw the contours and the reference image. All patients underwent daily MRI scans for setup verification prior to treatment during the treatment period. Of these, only weekly MRI images were included in the study. All MRIs (six MRI sets per patient) were acquired on a $0.35 \mathrm{~T}$ ViewRay scanner system (ViewRay Inc., Cleveland, OH, USA). A non-contrast true fast imaging with steady-state precession (TRUFI) sequence was used for all MRI scanning [41]. The MRI resolution was $1.5 \times 1.5 \times 3.0 \mathrm{~mm}^{3}$ with a typical imaging time of $25 \mathrm{~s}$ and a field of view of $54 \times$ $47 \times 43 \mathrm{~cm}^{3}$. The CTV was defined to include prostate and seminal vesicles. The planning target volume (PTV) was generated by adding margins of $3 \mathrm{~mm}$ and $5 \mathrm{~mm}$ in all directions to the prostate and seminal vesicles, respectively. The rectum and bladder were not controlled for filling. These structures (six RT structure sets per patient) were delineated on each MRI by a physician to ensure that the contouring practice was consistent. The prescription dose to the PTV was $70 \mathrm{~Gy}$ in 28 fractions for all patients. Two strategies for MRgRT plans were considered. One plan used the repositioning technique, which was called as the Soft plan. The repositioning technique finds the best matches of the target volume between the iMRI and weekly MRI (nMRI). Patient repositioning was performed by translational correction because of the motion limitations of the ViewRay couch. The other plan used the replanning technique for every nMRI, which was called as the Adaptive plan. Thus, each patient had one initial IMRT plan (iIMRT), five Soft plans, and five Adaptive plans.

\section{Techniques and treatment plans}

The iCT was deformed to iMRI to generate the deformed CT (dCT) for dose calculation. The initial structure set (iRS) contoured on iMRI was also included in the treatment planning. A total of 13 fields (five beam groups) were used to generate an iIMRT plan for each patient with the tri $-{ }^{60} \mathrm{Co}$ system. The gantry angles of the fields were $0^{\circ}$ (group 1); $24^{\circ}, 144^{\circ}$, and $264^{\circ}$ (group 2); $48^{\circ}, 168^{\circ}$, and $267^{\circ}$ (group 3); $72^{\circ}, 192^{\circ}$, and $312^{\circ}$ (group 4); and $96^{\circ}, 216^{\circ}$, and $336^{\circ}$ (group 5). The values for the IMRT efficiency that smoothed the fluence map intensity pattern (could be set from 0 to 1 ) and maximized the deliverable beam-on segments per field were 0.5 and 10 , respectively. The optimization and dose calculation were performed with an imaging surface coil in the presence of a magnetic field and using a calculation grid of $3 \mathrm{~mm}$. The Monte Carlo calculation algorithm developed by the manufacturer (ViewRay Inc., Cleveland, $\mathrm{OH}$ ) was used to calculate the dose. The number of histories in the system was set to $2.4 \times 10^{6}$. This stetting achieves about $1 \%$ statistical uncertainty for a dose grid of $3 \mathrm{~mm}$ cubed voxels. The optimization was performed according to National Comprehensive Cancer Network (NCCN) guidelines, and each plan was normalized to cover $100 \%$ of the PTV with $95 \%$ of the prescription dose. The MRIdian $^{\text {TM }}$ system use a convex nonlinear programing model for dose optimization. Table 2 shows the dose optimization parameters for the target and OARs. For a relatively fair comparison, all plans used these optimization parameters. Finally, the iIMRT plan was determined according to several beam delivery parameters. For each patient, six sets of MRIs (nMRI) and structure sets (nRS) were scanned during

Table 2 Summary of MRIdian ${ }^{\text {TM }}$ optimization parameters for target volume and OARs

\begin{tabular}{|c|c|c|c|c|c|c|c|}
\hline \multirow[t]{2}{*}{ Structure } & \multicolumn{2}{|c|}{ Importance } & \multicolumn{2}{|l|}{ Power } & \multirow{2}{*}{$\begin{array}{l}\text { Threshold } \\
\text { (Gy) }\end{array}$} & \multirow{2}{*}{$\begin{array}{l}\text { Prescription } \\
\text { (Gy) }\end{array}$} & \multirow[t]{2}{*}{ Offset } \\
\hline & Lower & Upper & Lower & Upper & & & \\
\hline Skin & - & 1 & - & 1 & 21 & - & \\
\hline Rectum & - & 1 & - & 1 & 56 & - & \\
\hline Bladder & - & 1 & - & 1 & 56 & - & \\
\hline apTV & 2 & 2 & 2 & 2 & - & 70 & 0.5 \\
\hline
\end{tabular}

aPTV planning target volume 
the treatment course and used in this study. For the Soft plan, the isocenter from iIMRT was relocated to the center of the PTV from nRS with nMRI. After the patient was repositioned based on target volume, the dose (from the iIMRT) was recalculated on nMRI with nRS. In contrast, the Adaptive plan was generated from the Soft plan with reoptimization by using the same constraints and associated weights for iIMRT. The d'CT was generated by deforming $\mathrm{dCT}$ to $\mathrm{nMRI}$ and used to calculate the dose for each plan. Each plan was compared at a prescription dose of $70 \mathrm{~Gy}$.

\section{Dose-volumetric evaluation and comparison}

The dose-volumetric parameters of the PTV, bladder, and rectum were calculated for all plans. The dosimetric index $\mathrm{D}_{\mathrm{x} \%}$ is defined as the dose to $\mathrm{x} \%$ of volume of a structure. For the PTV, the mean dose $\left(D_{\text {mean }}\right), D_{95 \%}$, $\mathrm{D}_{98 \%}$, and $\mathrm{D}_{2 \%}$ were calculated. The conformity index (CI), homogeneity index (HI), and gradient index (GI) were calculated as follows $[42,43]$ :

$$
\begin{aligned}
& \text { Conformity index }(C I)=\frac{\text { Volume of reference isodose }}{\text { Volume of target volume }} \\
& \text { Homogeneity index }(H I)=\frac{D_{2 \%}-D_{98 \%}}{D_{50 \%}} \\
& \text { Gradient index }(G I)=\frac{V_{50 \%} \text { of the prescription dose }}{V_{100 \%} \text { of the prescription dose }}
\end{aligned}
$$

For the bladder and rectum, $\mathrm{D}_{15 \%}, \mathrm{D}_{25 \%}, \mathrm{D}_{35 \%}$, and $\mathrm{D}_{50 \%}$ were calculated. During the treatment course, the PTV, bladder, and rectum were evaluated for changes in volume and their effect on the dosimetric parameters. Each technique was compared according to the abovementioned dose-volumetric parameters. Wilcoxon tests were performed at the $95 \%$ confidence level to examine the statistical significance of differences between the dose-volumetric parameters of the plans. The iIMRT (i.e., IMRT plan for the first fraction) was used as a criterion for comparing the differences. The given plan was defined as "reproducible" for both PTV and OARs, respectively, if the difference was less than 5\% compared to the iIMRT.

\section{Results}

Dose-volumetric evaluation and comparison of PTVs

Table 3 lists the results of dose-volumetric evaluation and a comparison of the PTVs for each plan. There were significant differences in $\mathrm{D}_{98 \%}, \mathrm{D}_{95 \%}$, and $\mathrm{D}_{2 \%}$ in PTV between the Soft and Adaptive plans $(p<0.05)$, except for $\mathrm{D}_{\text {mean }}$. For each dosimetric index, one plan group including the iIMRT exhibited an average value relative to the prescription dose and its standard deviation (Mean \pm SD). With regard to the index related to target coverage, the Adaptive plan clearly demonstrated superior results for $\mathrm{D}_{98 \%}(99.1 \pm 0.3 \%)$. For $\mathrm{D}_{95 \%}$, the average value for Adaptive plan was always $100 \%$ because each plan was normalized to cover $100 \%$ of the PTV with $95 \%$ of the prescription dose. The difference was statistically significant compared with that of the Soft plan $(p=0.011)$. Compared with iIMRT, the average difference for the Adaptive plan was less than $1 \%$ for all dose-volumetric evaluations. The percentage of reproducible plans for $\mathrm{D}_{98 \%}$ was $77.9 \%$ with the Soft plan.

Figure 1 shows the averaged dose-volumetric values of the PTV for each plan and all patients during treatment. The target coverage during the treatment course did change significantly with the Soft plan. There was no significant change in $D_{\max }$ and $\mathrm{D}_{\text {mean }}$ as the treatment progressed with all plans. Figure 2 shows the averaged $\mathrm{CI}, \mathrm{HI}$, and GI of the PTV for all plans during treatment. The average $\mathrm{CI}$ values during the treatment course were $1.38 \pm 0.27$ with the Soft plan, and significant changes were observed during the treatment course. In this analysis, the IIMRT was excluded because this was the reference plan. The CI value was lower in the Adaptive plan but not statistically significant compared to that in the Soft plans $(p=0.08)$. However, HI and GI values were also lower in the Adaptive plan and statistically significant, compared to the Soft plans $(p<0.05)$. The averaged

\begin{tabular}{|c|c|c|c|c|c|}
\hline Indices & Plan & Mean \pm SD (\%) & $p$-value & $\%$ of reproducible plan ${ }^{a}$ & Average difference (\%) \\
\hline \multirow[t]{2}{*}{$D_{\text {mean }}$} & Soft & $102.5 \pm 0.6$ & 0.099 & 100 & $0.1 \pm 0.9$ \\
\hline & Adaptive & $102.2 \pm 0.3$ & & 100 & $-0.1 \pm 0.5$ \\
\hline \multirow[t]{2}{*}{$D_{98 \%}$} & Soft & $96.6 \pm 3.3$ & $0.005^{*}$ & 77.9 & $-2.8 \pm 4.1$ \\
\hline & Adaptive & $99.1 \pm 0.3$ & & 100 & $-0.2 \pm 0.6$ \\
\hline \multirow[t]{2}{*}{$D_{95 \%}$} & Soft & $98.5 \pm 2.6$ & $0.011^{*}$ & 92.6 & $-1.8 \pm 3.2$ \\
\hline & Adaptive & 100 & & 100 & 0 \\
\hline \multirow[t]{2}{*}{$D_{2 \%}$} & Soft & $105.5 \pm 1.0$ & $<0.001^{*}$ & 100 & $0.7 \pm 1.0$ \\
\hline & Adaptive & $104.6 \pm 0.4$ & & 100 & $-0.4 \pm 1.0$ \\
\hline
\end{tabular}
$\mathrm{HI}$ and GI values for the Soft plan compared to those of

Table 3 Dose-volumetric evaluation and comparison between PTVs during the treatment

*Statistically significant comparisons; ${ }^{\text {a }}$ Percentage of plans with a difference of $<5 \%$ compared to the initial plan 

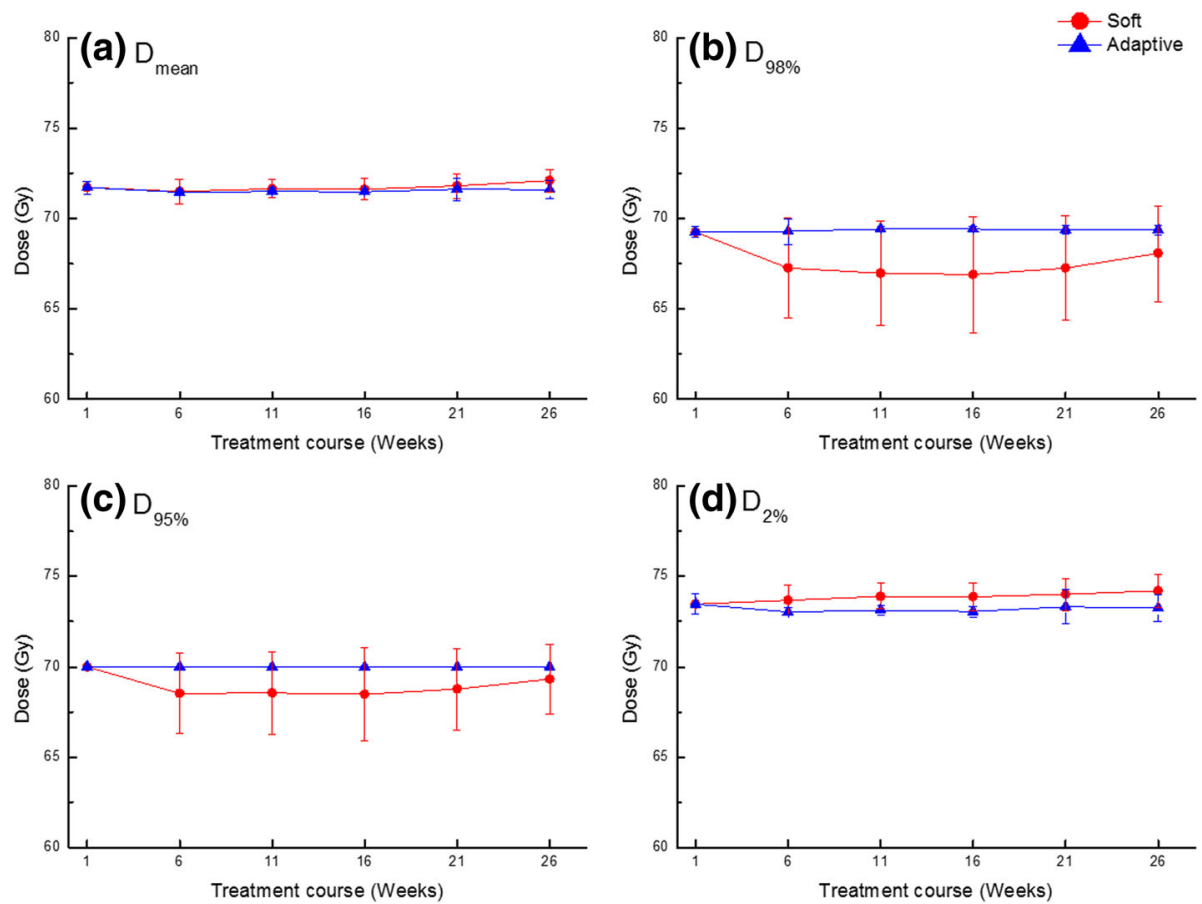

Fig. 1 Averaged dose-volumetric values of (a) $D_{\text {mean }}$ (b) $D_{98 \%}$ (c) $D_{95 \%}$ and (d) $D_{2 \%}$ for the target volume during the treatment course

iIMRT were $0.09 \pm 0.05$ and $1.11 \pm 0.18$, respectively. All indices for the Adaptive plan stayed the same compared to those of iIMRT during the treatment course; the average values of $\mathrm{CI}, \mathrm{HI}$, and GI were $1.28 \pm 0.22$, $0.06 \pm 0.01$, and $1.04 \pm 0.11$, respectively.
Dose-volumetric evaluation and comparison for the bladder Figure 3 shows the averaged dose-volumetric values of the bladder for each technique and all patients during treatment. With the Soft and Adaptive plans, the average $\mathrm{D}_{15 \%}$ values excluding that of iIMRT during the treatment
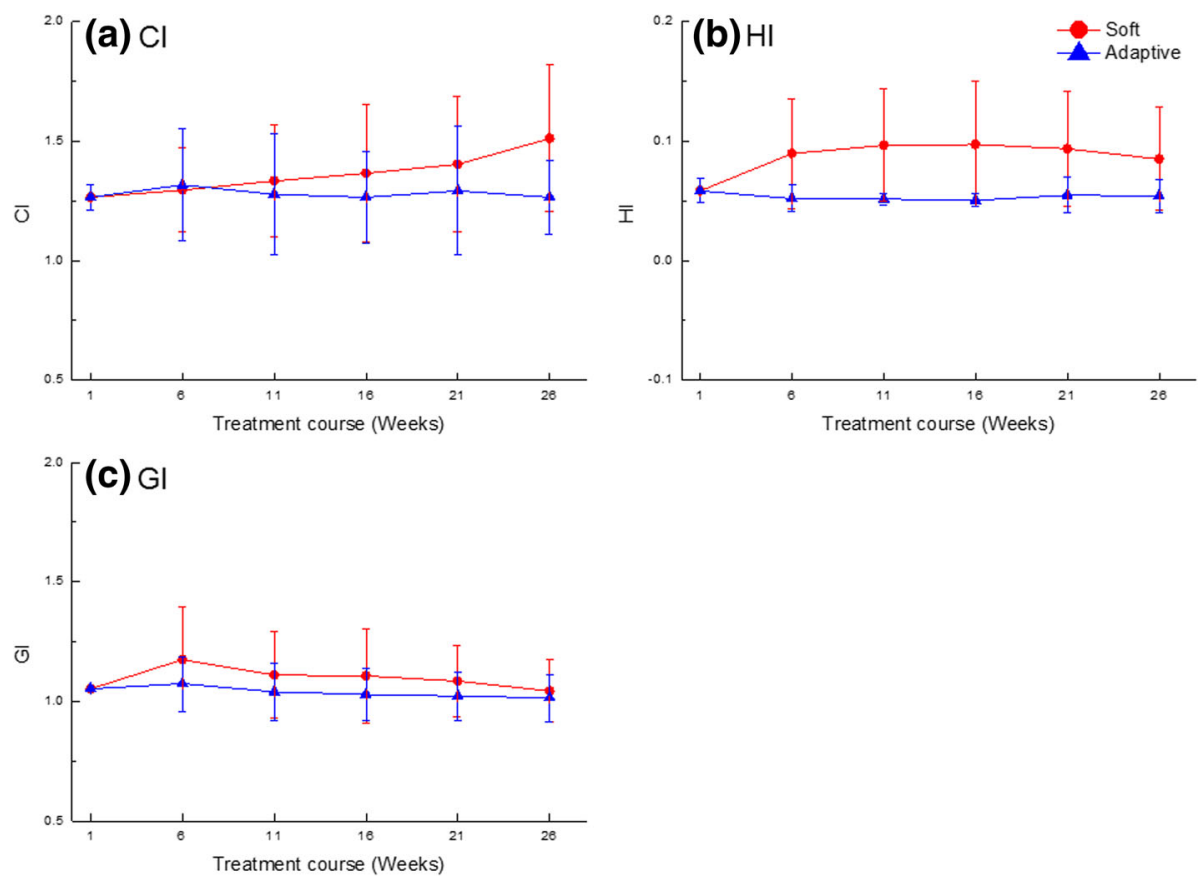

Fig. 2 Averaged dosimetric indices of $(\mathbf{a}) \mathrm{Cl},(\mathbf{b}) \mathrm{HI}$, and (c) $\mathrm{Gl}$ for the target volume during the treatment course 

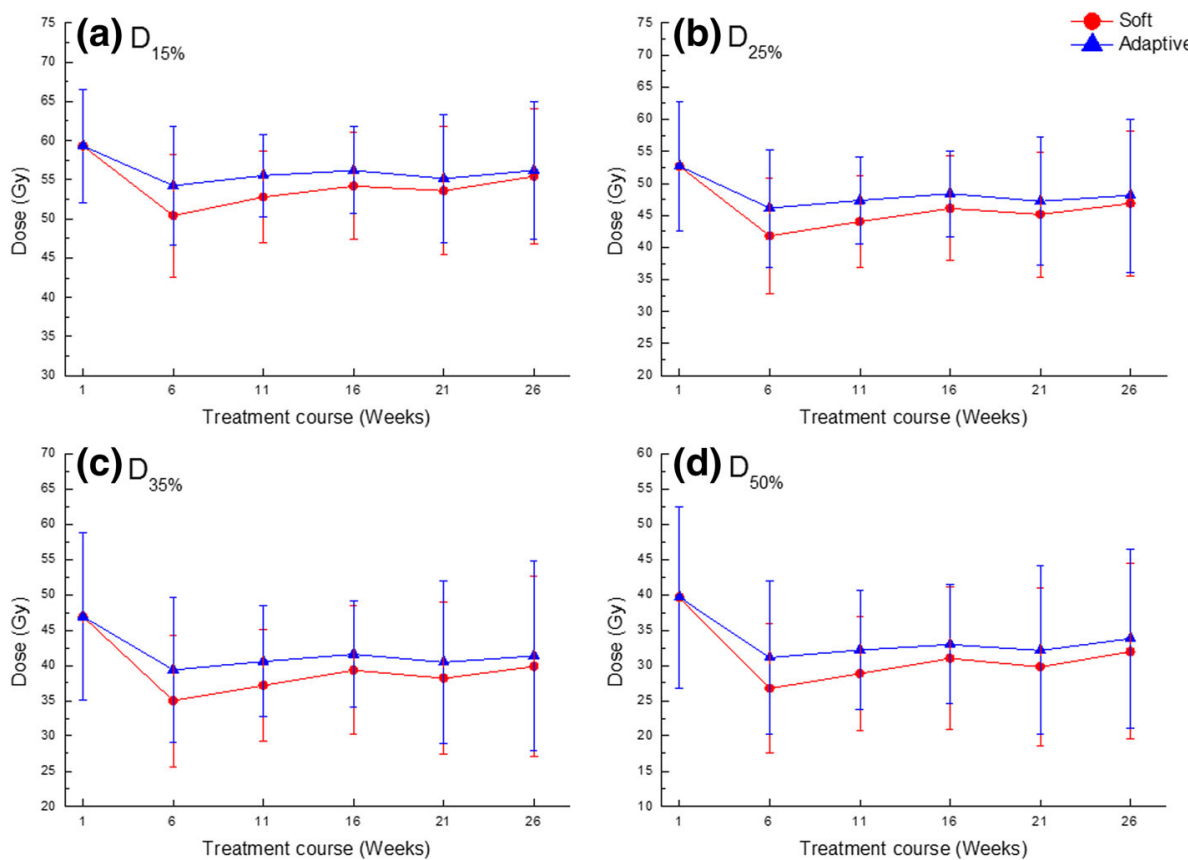

Fig. 3 Averaged dose-volumetric values of (a) $D_{15 \%}$ (b) $D_{25 \%}$ (c) $D_{35 \%}$ and (d) $D_{50 \%}$ for the bladder during the treatment course

course were $53.3 \pm 7.6$ and $55.5 \pm 7.1 \mathrm{~Gy}$, respectively. The average $\mathrm{D}_{25 \%}$ values were $44.8 \pm 9.2$ and $47.4 \pm 9.0 \mathrm{~Gy}$, respectively. The average $D_{35 \%}$ values were $37.9 \pm 10.1$ and $40.7 \pm 10.2$ Gy, respectively. The average $D_{50 \%}$ values were $29.7 \pm 10.3$ and $32.5 \pm 10.4$ Gy, respectively. The Adaptive plan did not achieve a better result than the that using the Soft plan during the treatment course. However, all plans had similar values for the standard deviation and exhibited a tolerable dose distribution of the bladder. Table 4 lists the results of the dose-volumetric evaluation and compares the plans for the bladder. There were significant differences in $\mathrm{D}_{15 \%}, \mathrm{D}_{25 \%}, \mathrm{D}_{35 \%}$, and $\mathrm{D}_{50 \%}$ in the bladder between the Soft and the Adaptive plans. For each dosimetric index, one plan group including the iIMRT showed the average value relative to the prescription dose and its standard deviation (Mean $\pm \mathrm{SD}$ ). The Adaptive plan showed the worse dose sparing compared with the Soft plan for bladder according to each dosimetric index. The difference was statistically significant compared to that for the Soft plan $(p<0.05)$. Even though the Adaptive plan showed a higher dose in the bladder than the Soft plan, the average percentage of differences compared to iIMRT showed lower values than those of the Soft plan. However, the percentages of reproducible plans for $D_{15 \%}, D_{25 \%}$, and $D_{35 \%}$ were 35.8 , 28.4 , and $25.3 \%$, respectively, which were higher values than those of the Soft plan except for $\mathrm{D}_{50 \%}$.

Dose-volumetric evaluation and comparison for the rectum Figure 4 shows the averaged dose-volumetric values of the rectum for each technique and all patients during treatment. With the Soft and Adaptive plans, the average $\mathrm{D}_{15 \%}$ values excluding that of iIMRT during the treatment course were $62.4 \pm 3.5$ and $61.3 \pm 1.9 \mathrm{~Gy}$. The average $\mathrm{D}_{25 \%}$ values were $57.7 \pm 4.1$ and $56.5 \pm 2.2 \mathrm{~Gy}$; the average

Table 4 Dose-volumetric evaluation and comparison of plans for the bladder during the treatment course

\begin{tabular}{llllll}
\hline Indices & Plan & Mean \pm SD (\%) & $p$-value & \% of reproducible plan ${ }^{\text {a }}$ & Average difference $(\%)$ \\
\hline$D_{15 \%}$ & Soft & $77.6 \pm 7.8$ & $0.01^{*}$ & 33.7 & $-9.14 \pm 15.1$ \\
& Adaptive & $80.2 \pm 8.2$ & & 35.8 & $-5.4 \pm 14.8$ \\
$D_{25 \%}$ & Soft & $65.9 \pm 9.8$ & $0.01^{*}$ & 25.3 & $-12.5 \pm 23.5$ \\
& Adaptive & $69.0 \pm 10.6$ & & 28.4 & $-7.2 \pm 24.9$ \\
$D_{35 \%}$ & Soft & $56.3 \pm 11.2$ & $0.016^{*}$ & 23.2 & $-14.6 \pm 33.5$ \\
& Adaptive & $59.6 \pm 12.2$ & & 25.3 & $-8.0 \pm 37.4$ \\
$D_{50 \%}$ & Soft & $44.8 \pm 11.5$ & $0.02^{*}$ & 23.2 & $-16.4 \pm 48.5$ \\
& Adaptive & $48.1 \pm 12.7$ & & 20.0 & $-8.0 \pm 56.3$ \\
\hline
\end{tabular}

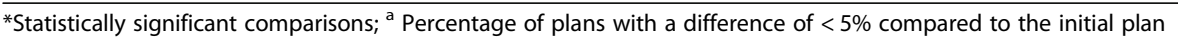



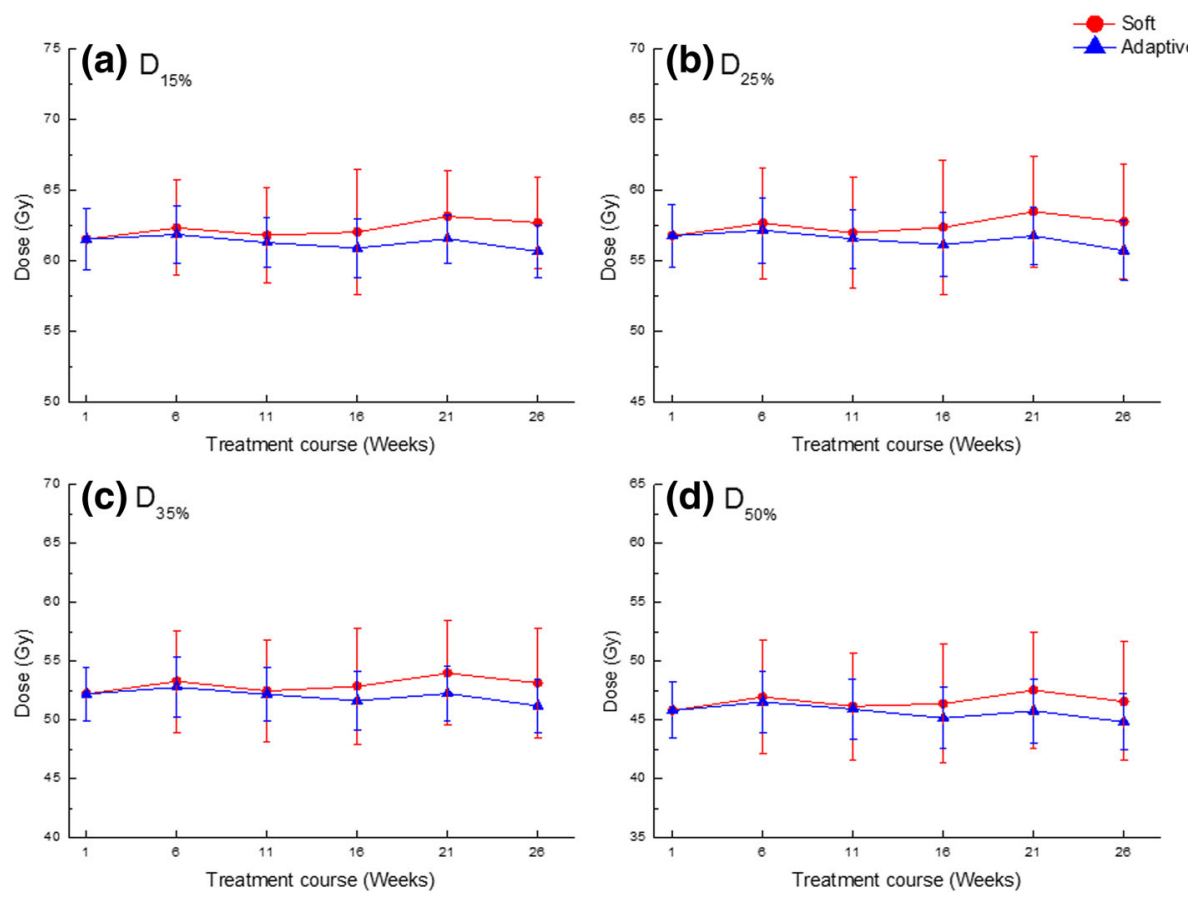

Fig. 4 Averaged dose-volumetric values of (a) $D_{15 \%}$ (b) $D_{25 \%}$, (c) $D_{35 \%}$ and (d) $D_{50 \%}$ for the rectum during the treatment course

$\mathrm{D}_{35 \%}$ values were $53.2 \pm 4.5$ and $52.0 \pm 5.4 \mathrm{~Gy}$; and the average $D_{50 \%}$ values were $46.7 \pm 4.8$ and $45.6 \pm 2.6$ Gy. All plans were below the tolerance dose of rectum. In contrast to the bladder, the Adaptive plan achieved better sparing than that by the Soft plan during the treatment course and showed the smallest standard deviation. Table 5 lists the results of the dose-volumetric evaluation and compares the plans for the rectum. The differences were only observed in $\mathrm{D}_{15 \%}$ and $\mathrm{D}_{35 \%}$ between the Soft and Adaptive plans, statistically $(p<0.05)$. For each dosimetric index, one plan group including iIMRT showed the average value relative to the prescription dose and its standard deviation (Mean $\pm \mathrm{SD}$ ). The Adaptive plan shows less average value and lower standard deviation for the rectum than the Soft plan according to each dosimetric index. However, no differences were observed between the plans in $\mathrm{D}_{25 \%}$ and $\mathrm{D}_{50 \%}(p>0.05)$. With the Soft plan, the percentages of reproducible plans for $\mathrm{D}_{15 \%}, \mathrm{D}_{25 \%}, \mathrm{D}_{35 \%}$ and $\mathrm{D}_{50 \%}$ were 87.4 , $87.4,87.4$, and $86.3 \%$, respectively. The average percentages of difference compared to iIMRT had positive mean values and large standard deviations: $1.1 \pm 6.4 \%, 1.1 \pm$ $7.7 \%, 1.2 \pm 8.8 \%$, and $1.2 \pm 9.9 \%$ for $\mathrm{D}_{15 \%}, \mathrm{D}_{25 \%}, \mathrm{D}_{35 \%}$ and $\mathrm{D}_{50 \%}$, respectively. With the Adaptive plan, on the other hand, the percentages of reproducible plans for $D_{15 \%}$, $\mathrm{D}_{25 \%}, \mathrm{D}_{35 \%}$ and $\mathrm{D}_{50 \%}$ were $89.5,86.3,81.1$, and $76.8 \%$, respectively. The average percentages of difference compared to iIMRT had negative mean values and small standard deviations: $-0.8 \pm 3.0 \%,-1.0 \pm 3.7 \%,-1.0 \pm$ $4.4 \%$, and $-1.1 \pm 5.8 \%$ for $\mathrm{D}_{15 \%}, \mathrm{D}_{25 \%}, \mathrm{D}_{35 \%}$ and $\mathrm{D}_{50 \%}$, respectively.

Table 5 Dose-volumetric evaluation and comparison of plans for the rectum during the treatment course

\begin{tabular}{llllll}
\hline Indices & Plan & Mean \pm SD (\%) & $p$-value & \% of reproducible plan ${ }^{\text {a }}$ & Average difference $(\%)$ \\
\hline$D_{15 \%}$ & Soft & $62.3 \pm 2.8$ & $0.009^{*}$ & 87.4 & $1.1 \pm 6.4$ \\
& Adaptive & $61.3 \pm 1.5$ & & 89.5 & $-0.8 \pm 3.0$ \\
$D_{25 \%}$ & Soft & $57.5 \pm 3.2$ & 0.16 & 87.4 & $1.1 \pm 7.7$ \\
& Adaptive & $56.5 \pm 1.8$ & & 86.3 & $-1.0 \pm 3.7$ \\
$D_{35 \%}$ & Soft & $75.7 \pm 5.1$ & $0.033^{*}$ & 87.4 & $1.2 \pm 8.8$ \\
& Adaptive & $74.4 \pm 2.7$ & & 81.1 & $-1.0 \pm 4.4$ \\
$D_{50 \%}$ & Soft & $66.5 \pm 5.6$ & 0.059 & 86.3 & $1.2 \pm 9.9$ \\
& Adaptive & $65.2 \pm 2.9$ & & 76.8 & $-1.1 \pm 5.8$
\end{tabular}

*Statistically significant comparisons; ${ }^{\text {a }}$ Percentage of plans with a difference of $<5 \%$ compared to the initial plan 


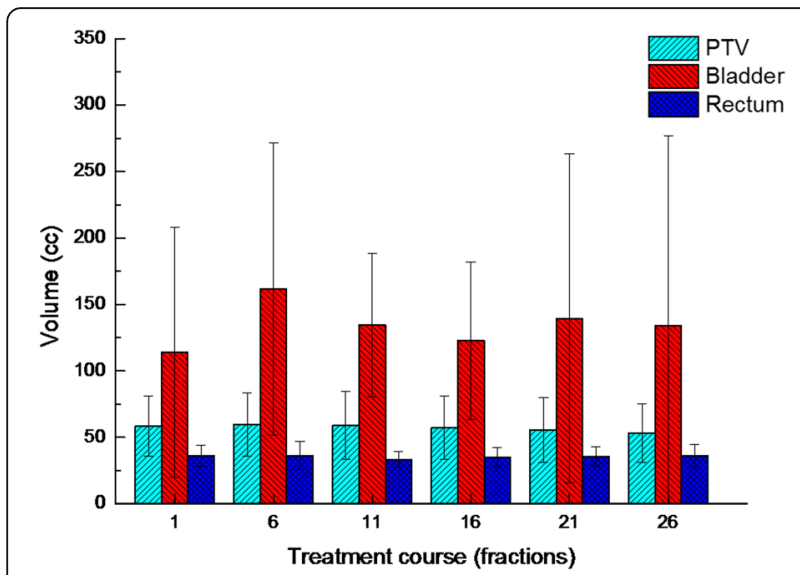

Fig. 5 Average volume variation of organs for all patients during the treatment course

\section{Discussion}

This study explored different MRgRT techniques to compensate for inter-fraction errors during prostate treatment. The Soft plan, which repositions the patient by using the target volume, may be sufficient for correcting anatomic variations. For OARs with the Soft plan, the dose to the bladder was relatively low, while the dose to the rectum was relatively consistent during treatment. The Adaptive plan, which is a replanning technique, may be suitable for correcting anatomic variations, and the doses to the OARs remained relatively consistent during treatment. The Adaptive plan performed very similarly to the initial plan and compensated for interfraction uncertainty. The large variations in the mean values and standard deviation indicate that substantial fluctuations in target coverage can occur weekly with IGRT techniques based on patient repositioning. Only the replanning technique (i.e., Adaptive plan) maintained a consistent quality during the treatment period in terms of target coverage. However, the Adaptive plan did not achieve the better results in terms of sparing the bladder during the treatment course. In this study, the enrolled patients were not controlled for bladder and rectum filling because they had been treated with gated radiotherapy using the ViewRay system. Thus, the bladder and rectum were filled with various volumes during the treatment course. Figure 5 shows the averaged volume variation for all patients during the treatment course. The changes in the bladder volume were significant with large standard deviations. Significant variations in the bladder volume affect both the bladder dose volume and positions of adjacent organs [44-46]. The Adaptive plan did achieve the better results for rectum sparing because the variation in the rectum volume was not significant. Figure 6 compares the example plans. In this case, the Soft plan had better OAR sparing than the Adaptive plan while missing target coverage. Thongphiew et al. used daily CBCT and also reported that the adaptive technique does not achieve the better OAR sparing for all fractions [18]. They discussed the limitations of CBCT. Compared to $\mathrm{CT}, \mathrm{CBCT}$ images make it more difficult to distinguish the boundaries of soft-tissue organs (e.g., the prostate, bladder, and rectum) because of more noise and poorer soft-tissue contrast. In this study, however, a $0.35 \mathrm{~T}$ MRI was used with high soft-tissue contrast and real-time imaging capability to exactly define the soft-tissue organ variations. Even low-field-strength MRI gives improved anatomic visualization compared with $\mathrm{CT}$ and $\mathrm{CBCT}$ [47]. The MRI allowed for the most accurate delineation of soft tissues and observation of organs changing. Thus, we adopted PTV margins of $3 \mathrm{~mm}$ and $5 \mathrm{~mm}$ in all directions for the prostate and seminal vesicles, respectively. McPartlin et al. reported that the CTVto-PTV margin can be safely reduced to less than 3 $\mathrm{mm}$ in MRI-guided prostate adaptive radiotherapy
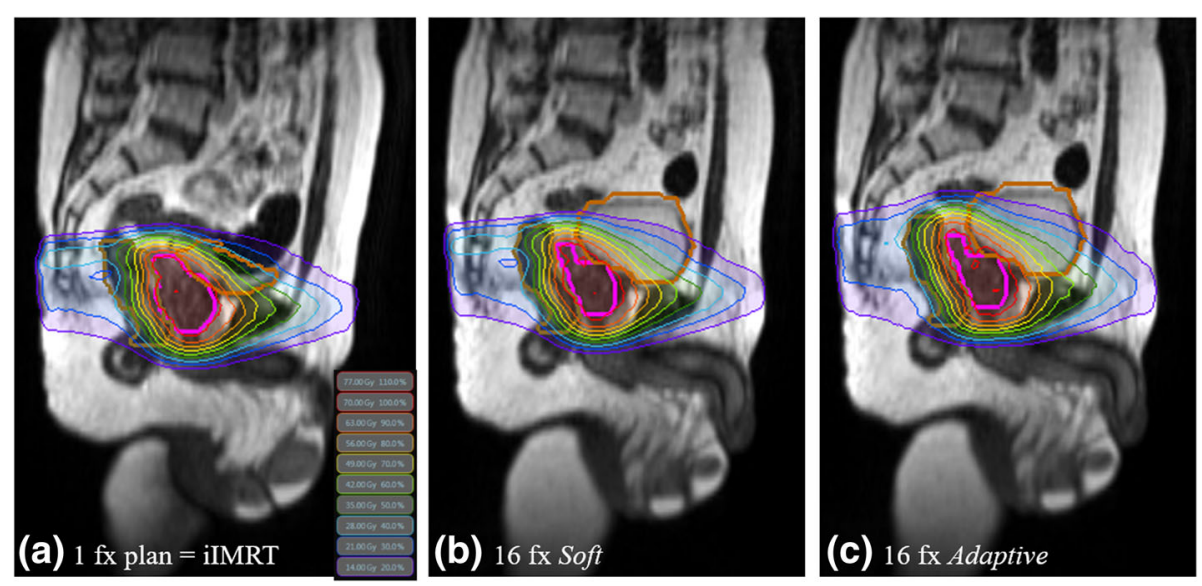

Fig. 6 Representative dose distributions with three plans: (a) initial, (b) Soft, and (c) Adaptive. All plans excluding the initial plan had the same organ structures and MRI 
because of high soft-tissue contrast and real-time imaging capability [48]. On the other hand, the plan quality was not better than that of a treatment-based linear accelerator (linac) because of the large penumbra of the ${ }^{60} \mathrm{Co}$ source and a large width of the multileaf collimator (MLC). The patient positioning techniques were also limited because only translational corrections were available. An MR-linac with $6 \mathrm{MV}$ beam is available in a clinic, and this system provides a better plan quality than a system equipped with ${ }^{60} \mathrm{Co}$ beams because of small penumbra and a small width of MLC $[49,50]$. In addition, adaptive planning will increase the time between the imaging and the beam delivery. The intra-fractional motion and deformation also increase with time [51]. These could be handled by using the gating delivery technique in treatment delivery. Furthermore, the Adaptive plan is not the actual dose delivered and the actual delivered dose should be assessed in parallel after treatment.

\section{Conclusions}

In this study, the MRgRT technique using adaptive planning was compared to the technique based on patient repositioning for 19 prostate cancer patient with the MRI. Patient repositioning based on the target volume (Soft plan) can relatively retain the target coverage for patients and the OARs remain at a clinically tolerance level during the treatment course. Although the Adaptive plan did not clinically improve for the dose delivered to OARs, it kept the dose delivered to the target volume constant. However, the Adaptive plan is beneficial when organ positions and volumes change considerably during treatment.

\begin{abstract}
Abbreviations
ADT: Androgen deprivation therapy; ART: Adaptive radiotherapy; CBCT: Conebeam computed tomography; Cl: Conformity index; CT: Computed tomography; CTV: Clinical target volume; dCT: Deformed CT; EBRT: External beam radiotherapy; GI: Gradient index; HI: Homogeneity index; iCT: Initial CT image; IGRT: Image-guided radiotherapy; IIMRT: Initial intensity-modulated radiation therapy plans; iMRI: Initial MRI; IMRT: Intensity-modulated radiation therapy; iRS: Initial structure set; kV: Kilovolt; Linac: Linear accelerator; MLC: Multileaf collimator; MRgRT: Magnetic resonance-guided radiation therapy; MRI: Magnetic resonance imaging; MV: Megavolt; NCCN: National comprehensive cancer network; nMRI: Weekly MRI; nRS: Weekly structure set; OARs: Organs at risk; PTV: Planning target volume; TRUFI: True fast imaging with steady-state precession
\end{abstract}

\section{Acknowledgments}

Not applicable.

\section{Authors' contributions}

$J I K$ and JMP conceived the study concept, compiled and analyzed the data, drafted the manuscript, and participated in all aspects of the study. $\mathrm{CHC}$ and HJA helped measure the data. YJK and JHK analyzed the data from a clinical perspective and engaged in discussions to improve the significance of this study. JHK oversaw and verified the study completion. All authors read and approved the final manuscript.

\section{Funding}

This research was supported by the National Research Foundation of Korea (NRF) grant funded by the Korea government (MSIP) (No.

2017M2A2A7A02020641 and 2017M2A2A7A02020643).

\section{Availability of data and materials}

Data sharing not applicable to this article because no datasets were generated or analyzed during the current study.

\section{Ethics approval and consent to participate}

An approval for this study was obtained from the institutional review board (IRB No. 1607-047-774).

\section{Consent for publication}

Not applicable.

\section{Competing interests}

The authors declare that they have no competing interests.

\section{Author details}

'Department of Radiation Oncology, Seoul National University Hospital, Seoul, Republic of Korea. ${ }^{2}$ Biomedical Research Institute, Seoul National University Hospital, Seoul, Republic of Korea. ${ }^{3}$ Institute of Radiation Medicine, Seoul National University Medical Research Center, Seoul, Republic of Korea. ${ }^{4}$ Center for Convergence Research on Robotics, Advanced Institutes of Convergence Technology, Suwon, Republic of Korea.

Received: 27 December 2018 Accepted: 26 July 2019

Published online: 06 August 2019

References

1. Daoud MA, Aboelnaga EM, Alashry MS, Fathy S, Aletreby MA. Clinical outcome and toxicity evaluation of simultaneous integrated boost pelvic IMRT/NMAT at different dose levels combined with androgen deprivation therapy in prostate cancer patients. Onco Targets Ther. 2017;10:4981-8.

2. Quan EM, Li X, Li Y, Wang X, Kudchadker RJ, Johnson JL, et al. A comprehensive comparison of IMRT and VMAT plan quality for prostate cancer treatment. Int J Radiat Oncol Biol Phys. 2012;83:1169-78.

3. Pasler M, Georg D, Wirtz H, Lutterbach J. Effect of photon-beam energy on VMAT and IMRT treatment plan quality and dosimetric accuracy for advanced prostate cancer. Strahlenther Onkol. 2011;187:792-8.

4. Davidson MT, Blake SJ, Batchelar DL, Cheung P. Mah K. Assessing the role of volumetric modulated arc therapy (VMAT) relative to IMRT and helical tomotherapy in the management of localized, locally advanced, and postoperative prostate cancer. Int J Radiat Oncol Biol Phys. 2011;80:1550-8.

5. Tsai CL, Wu JK, Chao HL, Tsai YC, Cheng JC. Treatment and dosimetric advantages between VMAT, IMRT, and helical tomotherapy in prostate cancer. Med Dosim. 2011;36:264-71.

6. Wolff D, Stieler F, Welzel G, Lorenz F, Abo-Madyan Y, Mai S, et al. Volumetric modulated arc therapy (VMAT) vs. serial tomotherapy, step-and-shoot IMRT and 3D-conformal RT for treatment of prostate cancer. Radiother Oncol. 2009;93:226-33.

7. Guckenberger M, Lawrenz I, Flentje M. Moderately hypofractionated radiotherapy for localized prostate cancer: long-term outcome using IMRT and volumetric IGRT. Strahlenther Onkol. 2014;190:48-53.

8. Muller AC, Lutjens J, Alber M, Eckert F, Bamberg M, Schilling D, et al. Toxicity and outcome of pelvic IMRT for node-positive prostate cancer. Strahlenther Onkol. 2012;188:982-9.

9. Frank SJ, Dong L, Kudchadker RJ, De Crevoisier R, Lee AK, Cheung R, et al. Quantification of prostate and seminal vesicle interfraction variation during IMRT. Int J Radiat Oncol Biol Phys. 2008;71:813-20.

10. Little DJ, Dong L, Levy LB, Chandra A, Kuban DA. Use of portal images and BAT ultrasonography to measure setup error and organ motion for prostate IMRT: implications for treatment margins. Int J Radiat Oncol Biol Phys. 2003;56:1218-24.

11. Gill SK, Reddy K, Campbell N, Chen C, Pearson D. Determination of optimal PTV margin for patients receiving CBCT-guided prostate IMRT: comparative analysis based on CBCT dose calculation with four different margins. J Appl Clin Med Phys. 2015;16:252-62

12. Engels B, Soete G, Verellen D, Storme G. Conformal arc radiotherapy for prostate cancer: increased biochemical failure in patients with distended rectum on the planning computed tomogram despite image guidance by implanted markers. Int J Radiat Oncol Biol Phys. 2009:74:388-91.

13. Sanguineti G, Endres EJ, Parker BC, Bicquart C, Little M, Chen G, et al. Acute toxicity of whole-pelvis IMRT in 87 patients with localized prostate cancer. Acta Oncol. 2008;47:301-10. 
14. Groher M, Kopp P, Drerup M, Deutschmann H, Sedlmayer F, Wolf F. An IGRT margin concept for pelvic lymph nodes in high-risk prostate cancer. Strahlenther Onkol. 2017;193:750-5.

15. Mayyas E, Kim J, Kumar S, Liu C, Wen N, Movsas B, et al. A novel approach for evaluation of prostate deformation and associated dosimetric implications in IGRT of the prostate. Med Phys. 2014:41:091709.

16. Shi W, Li JG, Zlotecki RA, Yeung A, Newlin H, Palta J, et al. Evaluation of kV cone-beam ct performance for prostate IGRT: a comparison of automatic grey-value alignment to implanted fiducial-marker alignment. Am J Clin Oncol. 2011;34:16-21.

17. Barney BM, Lee RJ, Handrahan D, Welsh KT, Cook JT, Sause WT. Imageguided radiotherapy (IGRT) for prostate cancer comparing $\mathrm{kV}$ imaging of fiducial markers with cone beam computed tomography (CBCT). Int J Radiat Oncol Biol Phys. 2011;80:301-5.

18. Thongphiew D, Wu QJ, Lee WR, Chankong V, Yoo S, McMahon R, et al. Comparison of online IGRT techniques for prostate IMRT treatment: adaptive vs repositioning correction. Med Phys. 2009;36:1651-62.

19. Nairz O, Merz F, Deutschmann H, Kopp P, Scholler H, Zehentmayr F, et al. A strategy for the use of image-guided radiotherapy (IGRT) on linear accelerators and its impact on treatment margins for prostate cancer patients. Strahlenther Onkol. 2008;184:663-7.

20. Song WY, Schaly B, Bauman G, Battista JJ, Van Dyk J. Evaluation of imageguided radiation therapy (IGRT) technologies and their impact on the outcomes of hypofractionated prostate cancer treatments: a radiobiologic analysis. Int J Radiat Oncol Biol Phys. 2006;64:289-300.

21. Delpon G, Llagostera C, Le Blanc M, Rio E, Supiot S, Mahe MA, et al. Use of IGRT for prostate cancers (OBI-CBCT Varian, ExacTrac BrainLAB and MVCT TomoTherapy). Cancer Radiother. 2009;13:399-407.

22. Miszczyk L, Leszczynski W, Szczepanik K, Majewski W. Comparison of two image guided radiation therapy (IGRT) methods used for prostate cancer patients--CBCT and 2D-2D kV. Przegl Lek. 2008;65:315-20.

23. O'Neill AG, Jain S, Hounsell AR, O'Sullivan JM. Fiducial marker guided prostate radiotherapy: a review. Br J Radiol. 2016;89:20160296.

24. Li XA, Wu Q, Orton CG. Point/counterpoint. Online adaptive planning for prostate cancer radiotherapy is necessary and ready now. Med Phys. 2014;41:080601.

25. Thornqvist $S$, Hysing LB, Zolnay AG, Sohn M, Hoogeman MS, Muren LP, et al. Adaptive radiotherapy in locally advanced prostate cancer using a statistical deformable motion model. Acta Oncol. 2013;52:1423-9.

26. Haverkort MA, van de Kamer JB, Pieters BR, Koning CC, van Herk M, van Tienhoven $\mathrm{G}$. Adaptive margin radiotherapy for patients with prostate carcinoma: what's the benefit? Radiother Oncol. 2012;105:203-6.

27. Lei $Y$, Wu Q. A hybrid strategy of offline adaptive planning and online image guidance for prostate cancer radiotherapy. Phys Med Biol. 2010; $55: 2221-34$

28. Hilman S, Smith R, Masson S, Coomber H, Bahl A, Challapalli A, et al. Implementation of a Daily Transperineal Ultrasound System as Imageguided Radiotherapy for Prostate Cancer. Clin Oncol (R Coll Radiol). 2017;29:e49.

29. Maund IF, Benson RJ, Fairfoul J, Cook J, Huddart R, Poynter A. Image-guided radiotherapy of the prostate using daily CBCT: the feasibility and likely benefit of implementing a margin reduction. Br J Radiol. 2014;87:20140459.

30. Zhang YD, Wu CJ, Bao ML, Li H, Yan X, Liu XS, et al. New RESOLVE-based diffusional kurtosis imaging in MRI-visible prostate Cancer: effect of reduced $b$ value on image quality and diagnostic effectiveness. AJR Am J Roentgenol. 2016;207:330-8.

31. Barth BK, Cornelius A, Nanz D, Eberli D, Donati OF. Comparison of image quality and patient discomfort in prostate MRI: pelvic phased array coil vs. endorectal coil. Abdom Radiol (NY). 2016;41:2218-26.

32. Lutgendorf-Caucig C, Fotina I, Stock M, Potter R, Goldner G, Georg D. Feasibility of CBCT-based target and normal structure delineation in prostate cancer radiotherapy: multi-observer and image multi-modality study. Radiother Oncol. 2011;98:154-61.

33. Tenhunen M, Korhonen J, Kapanen M, Seppala T, Koivula L, Collan J, et al. MRI-only based radiation therapy of prostate cancer: workflow and early clinical experience. Acta Oncol. 2018:57:902-7.

34. Ghose S, Mitra J, Rivest-Henault D, Fazlollahi A, Stanwell P, Pichler P, et al. MRI-alone radiation therapy planning for prostate cancer: automatic fiducial marker detection. Med Phys. 2016;43:2218.

35. Arrayeh E, Westphalen AC, Kurhanewicz J, Roach M 3rd, Jung AJ, Carroll PR, et al. Does local recurrence of prostate cancer after radiation therapy occur at the site of primary tumor? Results of a longitudinal MRI and MRSI study. Int J Radiat Oncol Biol Phys. 2012;82:e787-93.

36. Pasquier D, Lartigau E. MRI prostate radiation therapy planning: when the patient distorts his own image (regarding Lambert et al., Radiother Oncol 2011; 98: 330-334). Radiother Oncol. 2012;102:163.

37. Mutic S, Dempsey JF. The ViewRay system: magnetic resonance-guided and controlled radiotherapy. Semin Radiat Oncol. 2014;24:196-9.

38. Iraha Y, Murayama S, Kamiya A, Iraha S, Ogawa K. Diffusion-weighted MRI and PSA correlations in patients with prostate cancer treated with radiation and hormonal therapy. Anticancer Res. 2012;32:4467-71.

39. Lambert J, Greer PB, Menk F, Patterson J, Parker J, Dahl K, et al. MRI-guided prostate radiation therapy planning: investigation of dosimetric accuracy of MRI-based dose planning. Radiother Oncol. 2011;98:330-4.

40. Sannazzari GL, Ragona R, Ruo Redda MG, Giglioli FR, Isolato G, Guarneri A. CT-MRI image fusion for delineation of volumes in three-dimensional conformal radiation therapy in the treatment of localized prostate cancer. $\mathrm{Br}$ J Radiol. 2002;75:603-7.

41. Marden FA, Wippold FJ 2nd, Perry A. Fast magnetic resonance imaging in steady-state precession (true FISP) in the prenatal diagnosis of a congenital brain teratoma. J Comput Assist Tomogr. 2003;27:427-30.

42. Hodapp N. The ICRU report 83: prescribing, recording and reporting photon-beam intensity-modulated radiation therapy (IMRT). Strahlenther Onkol. 2012;188:97-9.

43. Paddick I, Lippitz B. A simple dose gradient measurement tool to complement the conformity index. J Neurosurg. 2006;105(Suppl):194-201.

44. Son CH, Melotek JM, Liao C, Hubert G, Pelizzari CA, Eggener SE, et al. Bladder dose-volume parameters are associated with urinary incontinence after postoperative intensity modulated radiation therapy for prostate cancer. Pract Radiat Oncol. 2016;6:e179-e85.

45. Frizzell B, Lovato J, Foster J, Towers A, Lucas J, Able C. Impact of bladder volume on radiation dose to the rectum in the definitive treatment of prostate cancer. J Community Support Oncol. 2015;13:288-91.

46. Viswanathan AN, Yorke ED, Marks LB, Eifel PJ, Shipley WU. Radiation dose-volume effects of the urinary bladder. Int J Radiat Oncol Biol Phys. 2010;76:S116-22.

47. Pathmanathan AU, van As NJ, Kerkmeijer LGW, Christodouleas J, Lawton CAF, Vesprini $D$, et al. Magnetic resonance imaging-guided adaptive radiation therapy: a "game changer" for prostate treatment? Int J Radiat Oncol Biol Phys. 2018;100:361-73.

48. McPartlin AJ, Li XA, Kershaw LE, Heide U, Kerkmeijer L, Lawton C, et al. MRIguided prostate adaptive radiotherapy - a systematic review. Radiother Oncol. 2016:119:371-80.

49. Park JM, Wu HG, Kim HJ, Choi CH, Kim Jl. Comparison of treatment plans between IMRT with MR-linac and VMAT for lung SABR. Radiat Oncol. 2019;14:105

50. Winkel D, Bol GH, Kiekebosch IH, Van Asselen B, Kroon PS, JurgenliemkSchulz IM, et al. Evaluation of Online Plan Adaptation Strategies for the 1.5T MR-linac Based on "First-In-Man" Treatments. Cureus. 2018;10:e2431.

51. Polat B, Guenther I, Wilbert J, Goebel J, Sweeney RA, Flentje M, et al. Intrafractional uncertainties in image-guided intensity-modulated radiotherapy (IMRT) of prostate cancer. Strahlenther Onkol. 2008;184:668-73.

\section{Publisher's Note}

Springer Nature remains neutral with regard to jurisdictional claims in published maps and institutional affiliations.

Ready to submit your research? Choose BMC and benefit from:

- fast, convenient online submission

- thorough peer review by experienced researchers in your field

- rapid publication on acceptance

- support for research data, including large and complex data types

- gold Open Access which fosters wider collaboration and increased citations

- maximum visibility for your research: over $100 \mathrm{M}$ website views per year

At $\mathrm{BMC}$, research is always in progress.

Learn more biomedcentral.com/submission 